\title{
Re-evaluation of Pasteuria ramosa Metchnik off 1888, a Bacterium Pathogenic for Daphnia Species
}

\author{
PETER HIRSCH
}

Institut für Allgemeine Mikrobiologie der Universität Kiel, West Germany

\begin{abstract}
Recent examination of Daphnia spp. collected from Lake Erie and several locations in southern Michigan, U.S.A., revealed the presence of structures that resembled those described by Metchnikoff in 1888 as stages of the life cycle of Pasteuria ramosa. Flat, elongated forms from the body cavity near the joints of extremities resembled, especially when aggregated in the form of rosettes, bundles of flat crystals rather than stages of a microorganism. Spherical or pear-shaped bodies with buds were found aggregated on the surface or inside of antennae and other extremities. Similarity of these bodies with certain budding bacteria is suggested, and it is proposed to retain the original name $P$. ramosa Metchnikoff but to restrict it to these budding forms only. The longitudinal fission attributed to this bacterium is dubious and may have resulted from misunderstanding crystal-like structures for microorganisms.
\end{abstract}

Transverse fission is the process by which most known bacteria multiply; a few forms produce buds. However, in 1888 Pasteuria ramosa was described by Metchnikoff (2) as multiplying by longitudinal fission as well as by budding. Longitudinal fission was surmised after observing what appeared to be side branches. The importance of this claim was well recognized by Metchnikoff, who proposed a special genus for these organisms among the bacteria.

Longitudinal fission in Pasteuria has not been observed since. Moreover, Metchnikoff's description also contained observations on lightrefractile bodies produced by Pasteuria and thought to be endospores. Henrici and Johnson (1) questioned the spore formation and pointed out that the spores might have been buds. The very complex life cycle of Pasteuria, ranging from rods with possible side branches to almost spherical forms which "expel spores," made this organism desirable for studies on bacterial morphogenesis.

During an investigation of Daphnia spp. from several habitats, certain structures were found which closely resembled those organisms described by Metchnikoff (2). This study resulted in findings which differed from those reported by Metchnikoff. Whereas he connected all the structures he had observed to create the organism's life cycle, it is now suggested that the first, rod-shaped stage with supposedly longitudinal fission be omitted because of its similarity with flat bundles of crystals. A superficial similarity with aggregates or colonies of actinomycetes is discussed.

\section{MATERIALS AND METHODS}

Daphnia material. A large sample of Daphnia spp. was kindly supplied by J. Winner of Windsor, Canada. The sample, labeled CR 5553, was collected on July 24, 1968, from Lake Erie as part of the Great Lakes studies; it was preserved with $10 \%$ formaldehyde. The daphniae appeared to be normal individuals in good health, with the possible exception of a few diseased forms. Other samples of Daphnia spp. were collected from Gull Lake in southern Michigan and around the Michigan State University Kellogg Biological Station, Hickory Corners, Mich. Finally, some specimens were kindly supplied by J. Green of Westfield College, London, England. In this latter case, specimens were selected which showed a reddish discoloration due to attack by bacteria other than pasteuriae.

Preparation of the Daphnia material. Whole organisms were investigated at low magnification; antennae and other extremities were removed for observation at higher magnification, rinsed several times, and mounted in water, saline, oil, or on $2 \%$ water-agar.

Microscopy. A Zeiss phase photomicroscope was used throughout with neofluar optics and Kodak Plus-X film. Enlargements were printed with a Fotorite rapid developing device. 


\section{RESULTS}

Several hundred daphniae were investigated. Many of these (53 specimens) showed varying degrees of "infestation" with two types of structures resembling stages of the life cycle of $P$. ramosa as described and depicted by Metchnikoff: (i) spherical or pear-shaped bodies, single or aggregating, and apparently multiplying by a budding process (Fig. $1 \mathrm{~A}-\mathrm{E}$ );

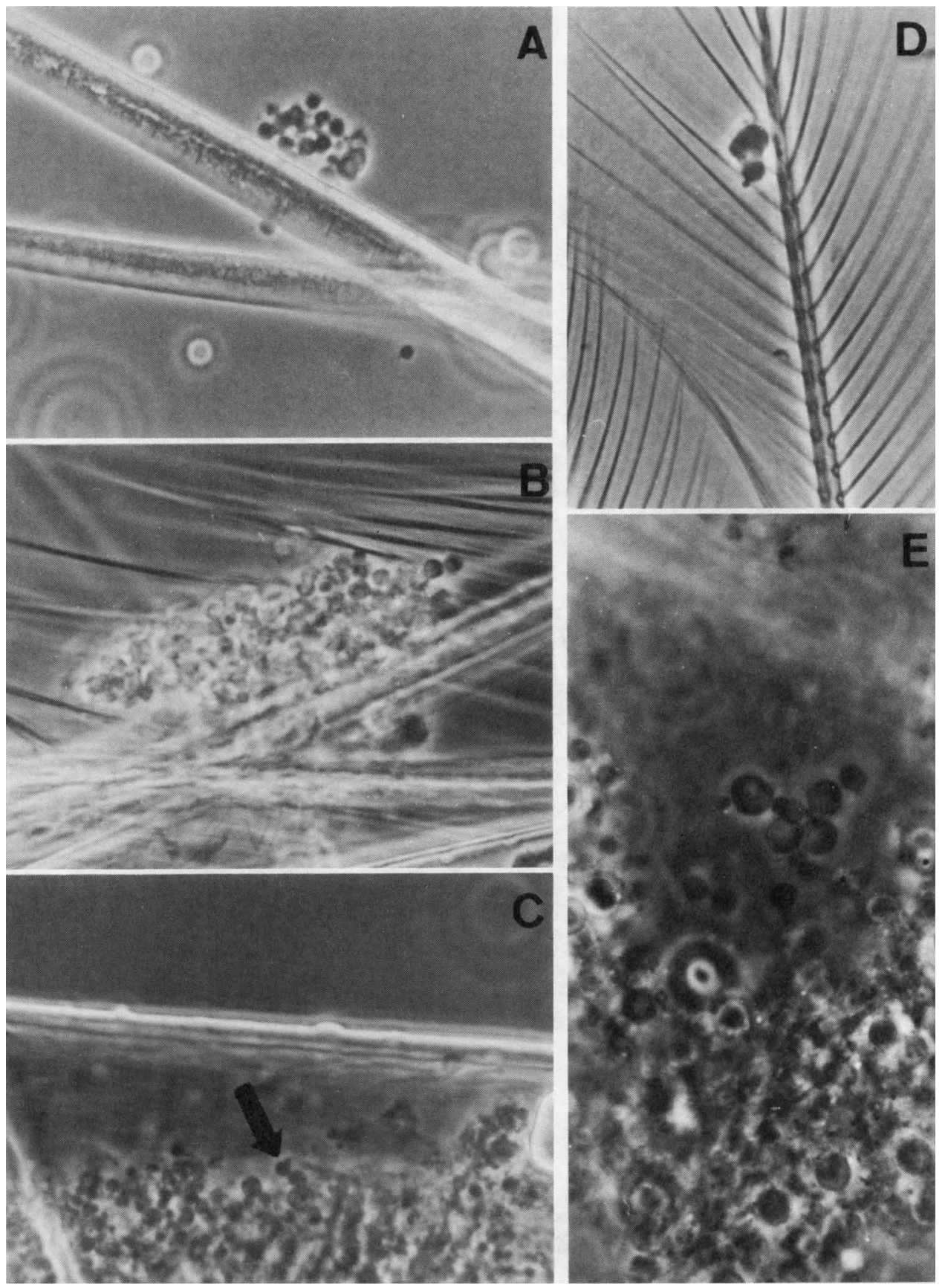

FIG. 1. Budding spheres in A, B, and D; antennae of Daphnia sp. from Lake Erie, in C and E. Magnification: $X$ 1,250 ; live, wet mounts on agar slides. 
and (ii) elongated, flat, rod or plate-shaped structures which were frequently aggregated in rosette form (Fig. $2 \mathrm{~A}-\mathrm{C}$ ). Whereas the former bodies were found frequently inside of the proximal portions of extremities as well as on their surface on the outside, the latter were mostly located inside of extremities near the joints. Both types were found together in the same individuals and only rarely appeared alone. The "budding" structures were often extremely numerous inside of the body cavity and were often found around or nearby muscle fibers. The flat, elongated structures were rare; many specimens contained only one aggregate. The rosettes were often tilted so that their flat shape could be recognized (Fig. 2A). The elongated structures had ends pointed at an angle, and often the edges appeared serrated (Fig. 2A). They thus strongly resembled aggregates of crystals similar to those formed by $\mathrm{Na}$ salts of fatty acids. Budding was never observed on these structures, and their form did not indicate any intermediate structure or shape with the budding spheres.

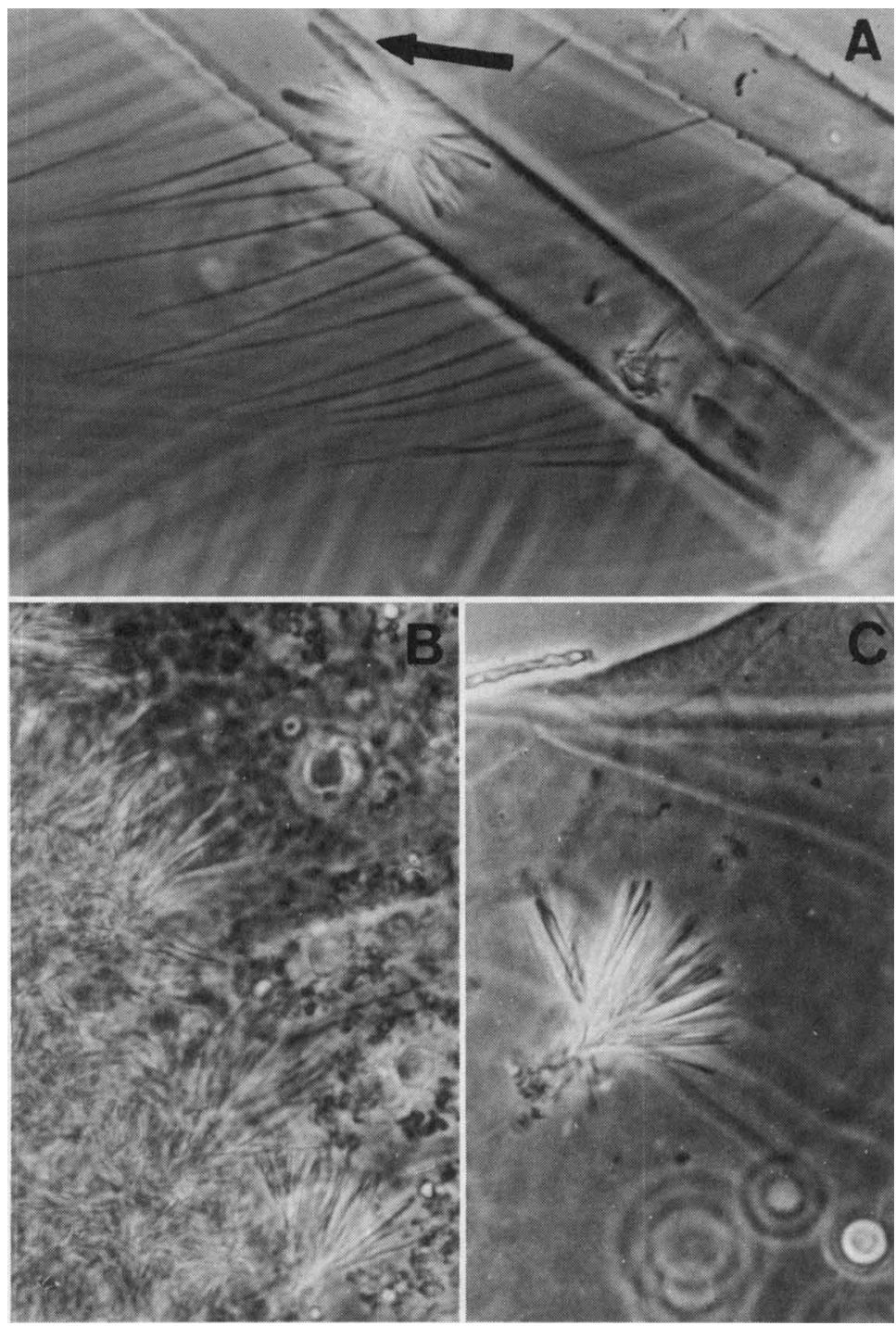

FIG. 2. Flat, longitudinal forms in antennae of Daphnia sp. from Lake Erie. These aggregates are believed to be crystals. Note serrated edge of the uppermost crystal in A. Magnification: $\times 1,250$; live, wet mounts on agar slides. 
The spherical or pear-shaped budding and aggregating forms varied in diameter from 1.5 to $5 \mu \mathrm{m}$; they did not contain anything resembling endospores. However, buds located laterally near the tip occasionally resembled those processes described by Metchnikoff as endospores.

The observed frequency of distribution and location of both types of structure were similar to those of specimens of different locations. No attempts were made to isolate and grow the two forms on artificial media.

\section{DISCUSSION}

To facilitate the discussion and interpretation of the observations, the life cycle of $P$. ramosa is reproduced here as shown by Metchnikoff (2) (Fig. 3). The similarity of stages 1 to 18 with the elongated forms or crystals shown in Fig. $2 \mathrm{~A}, \mathrm{~B}$, and $\mathrm{C}$ is evident, especially where these aggregate (stages 1-12). The stages supposedly multiply by longitudinal fission. Metchnikoff believed that he saw real side branches, but branching was not observed in the recent Daphnia material. The flat structures thought to be crystals appear to be branched when covering each other partly or when lying on the sides on top of each other.

A certain similarity of the flat structures to young actinomycete mycelia (Nocardia) is sometimes suggested, but flat, transparent "cells" of crystal shape have not been observed as yet in this group. Serration of the edges of elongated forms would also point to crystals. There does not appear to be a connection between the flat forms and the budding ones, other than the fact that they occur occasionally in the same diseased specimens.

Stages 19 to 21 do not resemble forms observed in this recent study. The "budding" forms were usually quite spherical with only a short, pointed (attached) pole. None of these stages is shown on the published micrographs, and they may thus be artifacts.

Stages 22 to 27 closely resemble the "budding" forms shown in Fig. 1 A to E. The photomicrograph (Fig. 1D) of Metchnikoff shows such an organism clearly, with the size being 3.3 by $4.2 \mathrm{~mm}$, which was calculated to be 3.9 by $5 \mu \mathrm{m}$. This compares favorably with the sizes measured in the recent material. A major difference is that aggregation is not pointed out by Metchnikoff in his drawings, whereas it is the rule for the presently described "budding" forms.

Brighter ends appearing in stages 22 to 27

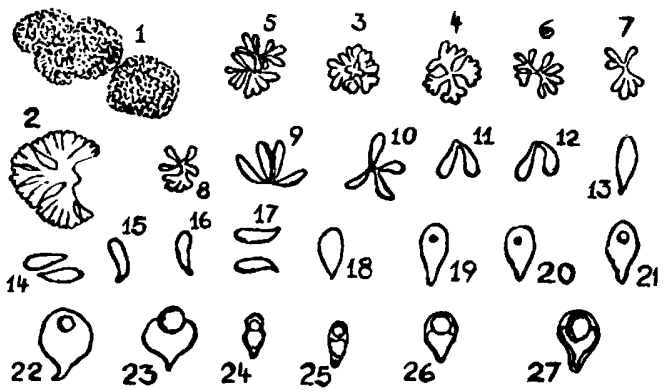

FIG. 3. The stages of the life cycle of Pasteuria ramosa from the original description by Metchnikoff (1888). Reproduced (redrawn) from plate 1 in reference 2 .

and increasing in brightness and size were interpreted by Metchnikoff as endospores; a stain was made specific for endospores. It was assumed, from the position of the small, spore-like bodies, that these were expelled upon maturity. No such process has ever been observed among other bacteria. Brighter areas similar in size, form, and location were observed in the recent material, but these were interpreted as areas of deoxyribonucleic acid and later on as perhaps buds. It has been suggested by Henrici and Johnson (1) that the endospore formation really might have been a budding process. Similar forms were also seen by J. T. Staley (personal communication; lake water) and by the author (unpublished data; lake water slides), in both cases in the absence of Daphnia organisms.

In conclusion, it is quite likely that the budding structures observed in and on Daphnia specimens in this present study were identical to those depicted and described by Metchnikoff in 1888 in his figures (stages) 22 to 27 . The question of whether these represent real organisms, perhaps pathogenic for Daphnia, cannot be answered at present without attempts at culturing them. The author recognizes the similarity of this organism to certain budding bacteria (Planctomyces) and suggests retaining the name Pasteuria ramosa for these stages only. It is thus proposed that $P$. ramosa Metchnik off 1888 be redefined: the organism in the new sense does not multiply by longitudinal fission but only by a budding process (stages 22 to 27 in the sense of Metchnikoff 1888). It is proposed that stages 1 to 21 be removed from the description as doubtful; these are possibly abiotic structures. Furthermore, it is suggested that the description contain the statement that endospore formation has not been observed in recent 
material, but that budding, resembling such a process, can be observed. Although the specific epithet ramosa now appears to be inappropriate in the name of this organism, this is not cause for changing it.

\section{ACKNOWLEDGMENTS}

Parts of this work were completed in the Department of Microbiology and Public Health, Michigan State University, East Lansing, Mich., with support from grant no. GB-7403 of the National Science Foundation and by an NSF institutional grant.
The skillful technical assistance of B. Sanders is gratefully acknowledged. The author also wishes to thank R. E. Buchanan, G. Lauff, J. T. Staley, and J. Winner for their interest and help during this study.

\section{LITERATURE CITED}

1. Henrici, A. T., and D. E. Johnson. 1935. Studies of freshwater bacteria. II. Stalked bacteria, a new order of Schizomycetes. J. Bacteriol. 30:61-92.

2. Metchnikoff, M. E. 1888. Pasteuria ramosa un représentant des bactéries à division longitudinale. Ann. Inst. Pasteur (Paris) 2: 165-170. 